\title{
Lamb Shift Approximation for Elements up to 80 in a Very Simple Way
}

\author{
Espen Gaarder Haug \\ Norwegian University of Life Sciences, Akershus, Norway \\ Email: espenhaug@mac.com
}

How to cite this paper: Haug, E.G. (2019) Lamb Shift Approximation for Elements up to 80 in a Very Simple Way. Advances in Materials Physics and Chemistry, 9, 234-237. https://doi.org/10.4236/ampc.2019.912018

Received: November 6, 2019

Accepted: December 8, 2019

Published: December 11, 2019

Copyright $\odot 2019$ by author(s) and Scientific Research Publishing Inc. This work is licensed under the Creative Commons Attribution International License (CC BY 4.0).

http://creativecommons.org/licenses/by/4.0/

\begin{abstract}
We suggest that the Lamb shift can be approximated by a very simple function that seems accurate enough for most experimental researchers working with elements where the relativistic effects of the electron are minimal, that is up to element 80 or so. Even if our new approximation does not show anything new in quantum chemistry per se, we think that it can be useful for experimental researchers and students of both quantum physics and chemistry; now everyone can calculate the Lamb shift on the back of an envelope.
\end{abstract}

\section{Keywords}

Lamb Shift, Hydrogen-Like Atom, Single Electron, Simple Approximation Formula

\section{A Very Simple Lamb Shift Approximation Formula}

The Lamb shift was discovered by Willis Lamb and measured for the first time in 1947 by Lamb and Retherford [1] [2] [3] on the hydrogen microwave spectrum. This shift is actually a difference in energy between two energy levels of the hydrogen atom that was not predicted by the Dirac [4] equation.

In this short note, we present a simple approximation for the Lamb shift for one electron in any atom. The only input needed in this formula is the atomic number. We compare it with far more complex methods presented in the literature and we provide a table of Lamb shift observations to show that our approximation is quite accurate.

We suggest that the Lamb shift can be approximated very well with the following function (the output will be in electron volts)

$$
E_{l} \approx \frac{X \times Z^{Y}}{100000},
$$


where $Z$ is the atomic number and $X$ and $Y$ are constants. Optimization indicates that setting $X=4.2$ and $Y=3.53$ gives accurate results. The optimizing is done on the criteria that the sum of the squared percentage error relative to the benchmark data ${ }^{1}$ is minimized, that is we are simply minimizing $\sum_{i=1}^{N}\left(\frac{z_{i}-f\left(z_{i}\right)}{z_{i}}\right)^{2}$. The method can be improved further by dividing the elements into segments and optimize $X$ and $Y$ for each segment. Another alternative is to add one more constant; this gives a function

$$
E_{l} \approx \frac{a+X \times Z^{Y}}{100000},
$$

where the optimized result based on the same optimization criteria as above gives $a=-1.3, X=4.49$ and $Y=3.5$.

Table 1 shows predictions from our two simple formulas compared to the predictions generated by much more complex calculations from quantum electrodynamics, see [5] and also [6] [7]. Our model gives minimal physical insight. It can best be described as a curve fitting function. However, we will claim that even simple curve fitting functions can be useful. If someone asks you about the Lamb shift in a job interview, for example, you can calculate it in your head, or at least on the back of an envelope. In addition, students with just an introductory course in physics and little or no knowledge of quantum electrodynamics can gain some basic intuition about the approximate size of the Lamb shift for a single electron.

Our approximation formula also provides good predictions for the higher elements. Recently, [8] measured the Lamb shift for hydrogen-like lead and gold using microcalorimeters. They measured $(211 \pm 42) \mathrm{eV}$ for hydrogen-like gold and $(260 \pm 53) \mathrm{eV}$ for hydrogen-like lead. Quantum electrodynamics predicts a Lamb shift of 205.2 for gold and 244.6 for lead. The Lamb shift prediction we get from our simplest approximation formula for hydrogen-like gold, element 79, is

$$
E_{l} \approx \frac{4.2 \times 79^{3.53}}{100000} \approx 209.83
$$

and for lead, element 82 , it is

$$
E_{l} \approx \frac{4.2 \times 82^{3.53}}{100000} \approx 239.34 .
$$

We conclude that our approximation seems quite accurate and may be sufficient for most experimenters. Our formula is very simple and could be useful for experimental researchers as well as students wanting to do quick back on the envelope Lamb shift calculations. However, for higher order elements (above 80 or so), relativistic effects start to emerge and the approximation is no longer accurate.

${ }^{1}$ In this case the Johnson and Soff data. 
Table 1. This Table shows our Lamb shift approximation as well as more accurate calculations based on quantum electrodynamics by Johnson and Soff. The outputs are in electron volts $(\mathrm{eV})$.

\begin{tabular}{|c|c|c|c|c|c|c|}
\hline Element & $\begin{array}{l}\text { Element } \\
\text { number }\end{array}$ & $\begin{array}{l}\text { Johnson } \\
\text { \& Soff }\end{array}$ & $\begin{array}{c}\text { Simple approx } \\
\qquad X \times Z^{Y}\end{array}$ & \% Error & $\begin{array}{l}\text { Simple approx } \\
\qquad a+X \times Z^{Y}\end{array}$ & $\%$ Error \\
\hline Hydrogen $\mathrm{H}$ & 1 & 0.000034 & 0.000042 & $24.3 \%$ & 0.000033 & $3.5 \%$ \\
\hline Helium He & 2 & 0.000445 & 0.000485 & $8.9 \%$ & 0.000495 & $11.1 \%$ \\
\hline Lithium Li & 3 & 0.001978 & 0.002030 & $2.6 \%$ & 0.002087 & $5.5 \%$ \\
\hline Beryllium Be & 4 & 0.005642 & 0.005604 & $0.7 \%$ & 0.005734 & $1.6 \%$ \\
\hline Boron B & 5 & 0.012638 & 0.012320 & $2.5 \%$ & 0.012537 & $0.8 \%$ \\
\hline Carbon C & 6 & 0.024326 & 0.023449 & $3.6 \%$ & 0.023743 & $2.4 \%$ \\
\hline Nitrogen N & 7 & 0.042180 & 0.040406 & $4.2 \%$ & 0.040733 & $3.4 \%$ \\
\hline Oxygen $\mathrm{O}$ & 8 & 0.067783 & 0.064738 & $4.5 \%$ & 0.065009 & $4.1 \%$ \\
\hline Fluorine $\mathrm{F}$ & 9 & 0.102797 & 0.098113 & $4.6 \%$ & 0.098183 & $4.5 \%$ \\
\hline Neon $\mathrm{Ne}$ & 10 & 0.148944 & 0.142315 & $4.5 \%$ & 0.141973 & $4.7 \%$ \\
\hline Sodium $\mathrm{Na}$ & 11 & 0.207925 & 0.199235 & $4.2 \%$ & 0.198195 & $4.7 \%$ \\
\hline Magnesium Mg & 12 & 0.281696 & 0.270869 & $3.8 \%$ & 0.268757 & $4.6 \%$ \\
\hline Aluminum Al & 13 & 0.371958 & 0.359310 & $3.4 \%$ & 0.355658 & $4.4 \%$ \\
\hline Silicon Si & 14 & 0.480818 & 0.466747 & $2.9 \%$ & 0.460980 & $4.1 \%$ \\
\hline Phosphorus P & 15 & 0.610135 & 0.595458 & $2.4 \%$ & 0.586889 & $3.8 \%$ \\
\hline Sulfur S & 16 & 0.762018 & 0.747813 & $1.9 \%$ & 0.735629 & $3.5 \%$ \\
\hline Chlorine $\mathrm{Cl}$ & 17 & 0.938326 & 0.926262 & $1.3 \%$ & 0.909518 & $3.1 \%$ \\
\hline Argon Ar & 18 & 1.141415 & 1.133343 & $0.7 \%$ & 1.110951 & $2.7 \%$ \\
\hline Potassium K & 19 & 1.372401 & 1.371670 & $0.1 \%$ & 1.342393 & $2.2 \%$ \\
\hline Calcium Ca & 20 & 1.634631 & 1.643937 & $0.6 \%$ & 1.606378 & $1.7 \%$ \\
\hline Scandium Sc & 21 & 1.929222 & 1.952915 & $1.2 \%$ & 1.905509 & $1.2 \%$ \\
\hline Titanium Ti & 22 & 2.260265 & 2.301449 & $1.8 \%$ & 2.242452 & $0.8 \%$ \\
\hline Vanadium V & 23 & 2.626024 & 2.692456 & $2.5 \%$ & 2.619942 & $0.2 \%$ \\
\hline Chromium Cr & 24 & 2.895073 & 3.128924 & $8.1 \%$ & 3.040772 & $5.0 \%$ \\
\hline Manganese Mn & 25 & 3.480287 & 3.613912 & $3.8 \%$ & 3.507800 & $0.8 \%$ \\
\hline Zinc Zn & 30 & 6.429914 & 6.878398 & $7.0 \%$ & 6.640028 & $3.3 \%$ \\
\hline Zirconium Zr & 40 & 16.874495 & 18.989829 & $12.5 \%$ & 18.174229 & $7.7 \%$ \\
\hline Tin Sn & 50 & 36.017933 & 41.745859 & $15.9 \%$ & 39.686355 & $10.2 \%$ \\
\hline Neodymium Nd & 60 & 68.365880 & 79.455361 & $16.2 \%$ & 75.123470 & $9.9 \%$ \\
\hline Ytterbium Yb & 70 & 122.832930 & 136.913216 & $11.5 \%$ & 128.851488 & $4.9 \%$ \\
\hline Mercury Hg & 80 & 217.843402 & 219.359751 & $0.7 \%$ & 205.618065 & $5.6 \%$ \\
\hline Thorium Th & 90 & 401.838626 & 332.449243 & $17.3 \%$ & 310.523953 & $22.7 \%$ \\
\hline Fermium Fm & 100 & 809.008650 & 482.224521 & $40.4 \%$ & 448.999987 & $44.5 \%$ \\
\hline Darmstadtium Ds & 110 & 1853.590700 & 675.095898 & $63.6 \%$ & 626.788082 & $66.2 \%$ \\
\hline
\end{tabular}

${ }^{2}$ The original Johnson and Soff values must be multiplied by 0.000123986 to get them into the unit system used here. 


\section{Summary}

We have presented some very simple approximation formulas for the Lamb shift for hydrogen-like atoms. The approximation seems to fall inside the error range of observed Lamb shifts and can, therefore, be an adequate approximation for practitioners. The formula also makes Lamb shift calculations available to a wider audience, including students with a limited math and physics background who are interested in gaining some intuitions on how large the Lamb shift is for hydrogen-like atoms.

\section{Acknowledgements}

Thanks to Victoria Terces for assisting with manuscript editing and thanks to Alan Lewis for useful comments on optimization criteria and thanks to an anonymous referee for useful comments.

\section{Conflicts of Interest}

The author declares no conflicts of interest regarding the publication of this paper.

\section{References}

[1] Lamb Jr., W.E. and Retherford, R.C. (1947) Fine Structure of the Hydrogen Atom by a Microwave Method. Physical Review, 72, 241. https://doi.org/10.1103/PhysRev.72.241

[2] Lamb Jr., W.E. and Retherford, R.C. (1950) Fine Structure of the Hydrogen Atom. Part I. Physical Review, 79, 549. https://doi.org/10.1103/PhysRev.79.549

[3] Lamb Jr., W.E. and Retherford, R.C. (1951) Fine Structure of the Hydrogen Atom. Part II. Physical Review, 81, 222. https://doi.org/10.1103/PhysRev.81.222

[4] Dirac, P.A.M. (1928) The Quantum Theory of the Electron. Proceedings of the Royal Society A, London, Vol. 117. https://doi.org/10.1098/rspa.1928.0023

[5] Johnson, W.R. and Soff, G. (1985) The Lamb Shift in Hydrogen-Like Atoms, $1 \leq z \leq 110$. Atomic Data and Nuclear Data Tables, 33, 405-446. https://doi.org/10.1016/0092-640X(85)90010-5

[6] Huff, R.W. (1969) Simplified Calculation of Lamb Shift Using Algebraic Techniques. Physical Review, 186, 1367. https://doi.org/10.1103/PhysRev.186.1367

[7] Lang, P.F. and Smith, B.C. (2013) Relativistic Corrections for Calculating Ionization Energies of One- to Five-Electron Isoelectronic Atomic Ions. ISRN Inorganic Chemistry, 2013, Article ID: 689040. https://doi.org/10.1155/2013/689040

[8] Kraft-Bermuth, S., Andrianov, V., Bleile, A., Echler, A., Egelhof, P., Grabitz, P., Ilieva, S., Kiselev, O., Kilbourne, C. and McCammon, D. (2017) Precise Determination of the 1s Lamb Shift in Hydrogen-Like Lead and Gold Using Microcalorimeters. Journal of Physics B: Atomic, Molecular, and Optical Physics, 50, 1-10. https://doi.org/10.1088/1361-6455/50/5/055603 